\title{
SCExAO: First Results and On-Sky Performance
}

\author{
Thayne Currie ${ }^{1}$, Olivier Guyon ${ }^{2}$, Frantz Martinache ${ }^{2}$, Christophe \\ Clergeon $^{2}$, Michael McElwain ${ }^{3}$, Christian Thalmann ${ }^{4}$, Nemanja \\ Jovanovic $^{2}$, Garima Singh ${ }^{2}$, and Tomoyuki Kudo ${ }^{2}$ \\ ${ }^{1}$ Department of Astronomy and Astrophysics, University of Toronto \\ email: currie@astro.utoronto.ca \\ ${ }^{2}$ National Astronomical Observatory of Japan \\ ${ }^{3}$ NASA-Goddard Space Flight Center \\ ${ }^{4}$ Astronomical Institute "Anton Pannekoek," University of Amsterdam
}

\begin{abstract}
We present new on-sky results for the Subaru Coronagraphic Extreme Adaptive Optics imager (SCExAO) verifying and quantifying the contrast gain enabled by key components: the closed-loop coronagraphic low-order wavefront sensor (CLOWFS) and focal plane wavefront control ("speckle nulling"). SCExAO will soon be coupled with a high-order, Pyramid wavefront sensor which will yield $>90 \%$ Strehl ratio and enable $10^{6}-10^{7}$ contrast at small angular separations allowing us to image gas giant planets at solar system scales. Upcoming instruments like VAMPIRES, FIRST, and CHARIS will expand SCExAO's science capabilities.
\end{abstract}

Keywords. instrumentation: adaptive optics, detectors, stars: planetary systems

\section{Introduction}

SCExAO is a next-generation extreme-AO platform designed to image and characterize the spectra of self-luminous jovian planets at solar system-like scales around nearby, young stars (Martinache and Guyon 2009). Components comprising SCExAO are being commissioned in several phases. Already in Phase 1 we have verified the performance of the Phase Induced Amplitude Apodization (PIAA) coronagraph, which improves our ability to image planets at $\approx 2 \lambda / \mathrm{D}$ and with full efficiency by better suppressing diffraction at the telescope pupil edge (Martinache et al. 2012a). Here, we describe on-sky verification of other key "Phase 1" components: the CLOWFS (Guyon et al. 2011), which corrects for tip-tilt errors to yield $\approx 10^{-3} \lambda / \mathrm{D}$ pointing accuracy, and "speckle nulling", which uses a deformable mirror (DM) to cancel out static speckles limiting planet detections at small separations. Finally, we describe the timeline for implementing SCExAO's high-order wavefront control and new instrumentation.

\section{Phase 1 Engineering Observations}

On November 14, 2012, we observed Pollux with SCExAO combined with the facility adaptive optics system (AO-188) and HiCIAO to test and verify the on-sky performance of the CLOWFS and speckle nulling components of SCExAO. After a close-loop sequence of speckle nulling, using images acquired by the SCExAO internal science camera (XS), we obtained a sequence of images acquired with HiCIAO alternating between the DM flat volt-map and the DM speckle nulling volt-map (e.g. Martinache et al. 2012b).

Figure 1 displays the combined image obtained without (left) and with (right) speckle nulling. Despite the XS's high read noise, the speckle nulling algorithm successfully (blindly) calibrated the brightest diffraction features of the long exposure image. For individual images, contrast gain is moderate $(\sim 2 \times$ for the brightest part of the first 

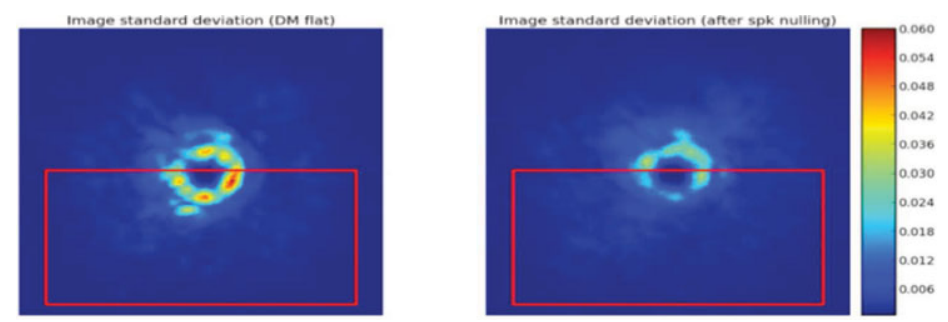

Figure 1. SCEXAO noise map of the combined image without (left) and with speckle nulling.

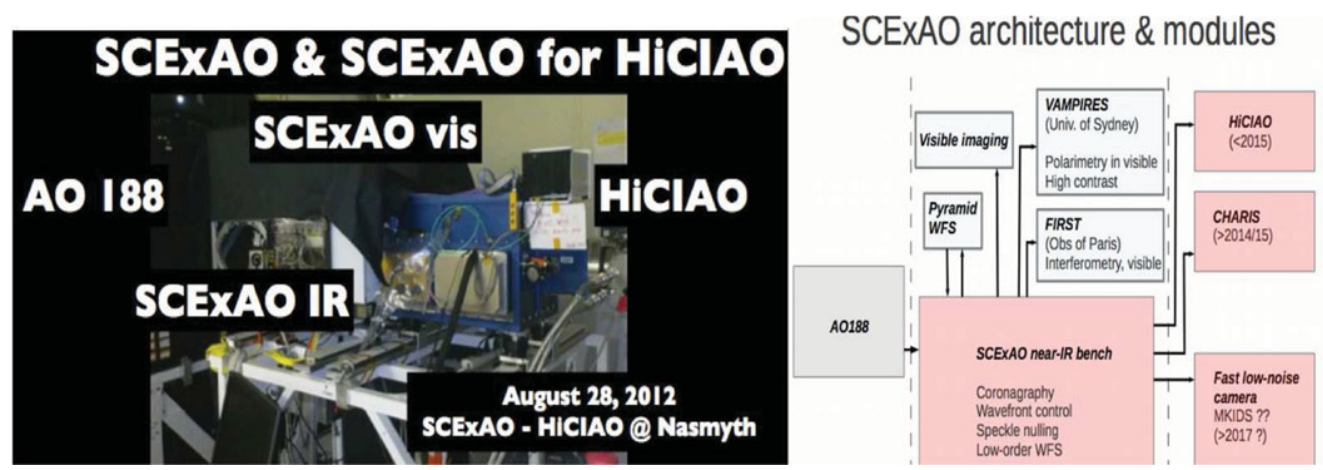

Figure 2. SCExAO as configured in Phase 1 (left) and Phase 2 (and beyond) (right).

ring), but real and persistent. Speckle nulling reduces the combined image's standard deviation over the entire frame by $\sim$ one-half. Inside the control region (boxed region), speckle nulling lowers the image standard deviation by about a factor of $\sim 7$. Thus, at the smallest angular separations $(2-4 \lambda / \mathrm{D})$, speckle nulling alone improves our detection limits by $\approx 1-2$ magnitudes (Martinache et al. 2013, in prep.).

\section{Current Timeline and Future Instrumentation}

SCExAO has now passed its Phase 1 requirements. Phase 2 of SCExAO commissioning in Summer/Fall 2013 will incorporate the 2000-actuator DM controlled by a high-order Pyramid wavefront sensor, which will yield $>90 \%$ Strehl at $1.6 \mu \mathrm{m}$ (Figure 2). Combined with PIAA; CLOWFS; "speckle nulling" calibrated from a faster, lower noise internal camera; and angular differential imaging plus aggressive post-processing (Marois et al. 2006, Currie et al. 2012); SCExAO should then achieve $10^{6}-10^{7}$ contrasts at $\approx 0.1-0.5$ " and thus image numerous young jovian planets at solar system-like scales. Interferometric instruments VAMPIRES and FIRST capable of studying planet formation at even smaller scales will be commissioned at the same time. Finally, CHARIS will eventually replace HiCIAO as the primary SCExAO high-contrast instrument and yield near-IR spectra of young planets (McElwain et al. 2013) to probe the planets' atmospheres.

\section{References}

Currie, T., et al., 2012, Ap. Lett., 760, L32

Guyon, O., et al., 2011, in Proc. SPIE, Vol. 8149

Marois, C., et al., 2006, Ap. J, 641, 556

Martinache, F., et al., 2009, in Proc. SPIE, Vol. 7440

Martinache, F., et al., 2012a, in Proc. SPIE, Vol. 8447

Martinache, F., et al., 2012b, PASP, 124, 1288

McElwain, M., et al., 2012, in Proc. SPIE, Vol. 8446 